\title{
The Ecology of Microbial Communities Associated with Macrocystis pyrifera
}

\author{
Vanessa K. Michelou ${ }^{1 *}$, J. Gregory Caporaso ${ }^{2,3}$, Rob Knight ${ }^{4,5}$, Stephen R. Palumbi ${ }^{1}$ \\ 1 Department of Biology, Hopkins Marine Station, Stanford University, Pacific Grove, California, United States of America, 2 Department of Biological Sciences, Northern \\ Arizona University, Flagstaff, Arizona, United States of America, 3 Argonne National Laboratory, Institute for Genomics and Systems Biology, Argonne, Illinois, United \\ States of America, 4 Department of Chemistry and Biochemistry, University of Colorado, Boulder, Colorado, United States of America, 5 Howard Hughes Medical Institute, \\ Boulder, Colorado, United States of America
}

\begin{abstract}
Kelp forests are characterized by high biodiversity and productivity, and the cycling of kelp-produced carbon is a vital process in this ecosystem. Although bacteria are assumed to play a major role in kelp forest carbon cycling, knowledge of the composition and diversity of these bacterial communities is lacking. Bacterial communities on the surface of Macrocystis pyrifera and adjacent seawater were sampled at the Hopkins Marine Station in Monterey Bay, CA, and further studied using 454-tag pyrosequencing of $16 \mathrm{~S}$ RNA genes. Our results suggest that $M$. pyrifera-dominated kelp forests harbor distinct microbial communities that vary temporally. The distribution of sequence tags assigned to Gammaproteobacteria, Alphaproteobacteria and Bacteriodetes differed between the surface of the kelp and the surrounding water. Several abundant Rhodobacteraceae, uncultivated Gammaproteobacteria and Bacteriodetes-associated tags displayed considerable temporal variation, often with similar trends in the seawater and the surface of the kelp. Bacterial community structure and membership correlated with the kelp surface serving as host, and varied over time. Several kelp-specific taxa were highly similar to other bacteria known to either prevent the colonization of eukaryotic larvae or exhibit antibacterial activities. Some of these kelp-specific bacterial associations might play an important role for $M$. pyrifera. This study provides the first assessment of the diversity and phylogenetic profile of the bacterial communities associated with $M$. pyrifera.
\end{abstract}

Citation: Michelou VK, Caporaso JG, Knight R, Palumbi SR (2013) The Ecology of Microbial Communities Associated with Macrocystis pyrifera. PLoS ONE 8(6): e67480. doi:10.1371/journal.pone.0067480

Editor: Tilmann Harder, University of New South Wales, Australia

Received March 4, 2013; Accepted May 19, 2013; Published June 19, 2013

Copyright: (C) 2013 Michelou et al. This is an open-access article distributed under the terms of the Creative Commons Attribution License, which permits unrestricted use, distribution, and reproduction in any medium, provided the original author and source are credited.

Funding: This work was supported by the David and Lucile Packard Foundation and by Stanford University, and by the Howard Hughes Medical Institute. The funders had no role in study design, data collection and analysis, decision to publish, or preparation of the manuscript.

Competing Interests: The authors have declared that no competing interests exist.

* E-mail: michelou@hawaii.edu

\section{Introduction}

Most aquatic organisms, particularly primary producers such as macroalgae, interact with their environment through their surface [1]. Bacteria-alga interactions vary from symbiotic to parasitic relationships that mainly depend on environmental parameters, such as the availability of inorganic nutrients and organic matter. In eutrophic coastal marine systems rapid bacterial biofilm growth on available surfaces takes place. This bacterial colonization is especially rapid if the surface is a potential source of nutrients, such as polysaccharides in exudates of kelp that may serve as source of carbon for heterotrophic bacteria living on the kelp surface [2,3]. Kelp exudates may shape bacterial community composition [4], and create communities that are kelp-specific rather than randomly assembled from the surrounding seawater [5-7].

The giant kelps are important foundation species that can perform essential roles in coastal kelp forest ecosystems, and represent an important natural resource, providing shelter and a growth substrate for many species of fish, invertebrates, other seaweeds and even microbial biofilms on their surface [8]. Kelp forests along the central coast of California are of tremendous importance for coastal biodiversity, productivity, and the human economy in the region, and the bacteria associated with their surfaces are believed to be important in carbon and nitrogen turnover in kelp forest food webs $[9,10]$. Recently, cultivation-independent methods have shed light on kelp-surface-associated microorganisms from kelps of the genera Laminaria and Saccharina [11,12]. Bacterial communities associated with different structural features of the kelp differ in composition, and these communities differ seasonally and geographically [11]. However, the ecology of microbial communities on the predominant kelp found along the western California coast, Macrocystis pyrifera, has not yet been investigated. For example, it is not known which taxa are present on $M$. pyrifera surfaces or how they compare with the surrounding environment.

This study reports an in-depth description of the diversity and phylogenetic association of the microbial communities associated with Macrocystis pyrifera. We compared microbial communities, using 454 sequencing of the bacterial 16S rRNA gene, from the surface of kelp and the surrounding water in Monterey Bay in March, April and May, to investigate the composition and temporal dynamics of kelp-associated bacterial communities. Our results revealed a distinct epiphytic microbial community associated with these macroalgae, which provides a foundation for understanding the microbial ecology of kelp forests.

\section{Methods}

Sample collection and DNA extraction

Samples of Macrocystis pyrifera were taken from the site of the Marine Life Observatory in Monterey Bay (Hopkins Marine 
Station) in March, April and May 2010. Duplicate kelp blades (lamina) were sampled from two different individual fronds adjacent to each other, from one-meter depth by scuba diving. Kelp blades were, removed with a knife and transferred into sterile plastic bags. In close proximity to the kelp (within $1 \mathrm{~m}$ distance), two water samples of one liter each were collected separately before kelp sampling, in sterile Duran bottles. Additional duplicate oneliter water samples were also collected outside the edge of the kelp forest approximately $1 \mathrm{~km}$ away, following the procedure described above. All water samples were kept at in situ water temperature $\left(13.1^{\circ} \mathrm{C}\right.$ in March, $14.4^{\circ} \mathrm{C}$ in April and $11.4^{\circ} \mathrm{C}$ in May) until immediate processing upon arrival in the laboratory, within one hour after sampling. Additional kelp and water samples were collected at ten meters depth in May. Only water samples from inside and outside the kelp forest perimeter were analyzed during April 2010 as the kelp samples from this time point failed to amplify.

Kelp blades were washed three times in sterile seawater to remove loosely associated bacterial cells. The lower part of the lamina (meristematic region) was sampled. Areas that were heavily epiphytized were avoided. Bacterial DNA was extracted from the surface of the kelp blades as described by [13], which leaves the algal host intact and extracts total DNA from the microbial community on the entire surface. Briefly, a surface of approximately $50 \mathrm{~cm}^{2}$ from each meristematic region of every individual kelp blade was placed into $100 \mathrm{ml}$ of calcium- and magnesium-free artificial seawater (CMFSW) containing $0.45 \mathrm{M} \mathrm{NaCl}, 10 \mathrm{mM}$ $\mathrm{KCl}, 7 \mathrm{mM} \mathrm{Na}_{2} \mathrm{SO}_{4}$, and $0.5 \mathrm{mM} \mathrm{NaHCO} 3$ and supplemented with $10 \mathrm{mM}$ EDTA and $1 \mathrm{ml}$ filter-sterilized rapid multienzyme cleaner (OSM low-foaming multienzyme detergent; Fisher cat. \#15-336-507) in $100 \mathrm{ml}$ Erlenmeyer flasks. Samples were then incubated for 2 hours at room temperature and $80 \mathrm{rpm}$ and then vortexed for 2 minutes. Kelp material was removed and the remaining liquid was centrifuged at $300 \times \mathrm{g}$ for $15 \mathrm{~min}$ to remove any remaining algal material. The supernatant was filtered onto a $0.2 \mu \mathrm{m}$ pore-sized Durapore filters and DNA was extracted from the filters by using a Cetyltrimethylammonium Bromide (CTAB) protocol involving two chloroform extractions and a high-salt isopropanol precipitation [14]. The extracted DNA was not pooled, and each sample represents the DNA from the surface community of a single kelp blade. Ten random segments of kelp (5 from before and 5 from after enzyme treatment) were stained with $5 \mu \mathrm{M}$ SYTO9 nucleic acid stain (Invitrogen, Carlsberg, CA) and examined by light microscopy to assess that the kelp tissues were intact without any visible lesions (data not shown).

Between 1.3 and 1.8 liters of seawater were vacuum filtered through $0.22-\mu \mathrm{m}$-pore-size Durapore membrane filters (Millipore) at the laboratory, with two replicates per sampling location. Filters were then preserved in a CTAB buffer and stored at $-80^{\circ} \mathrm{C}$ until DNA extractions were performed. DNA was extracted from the filters by using the same CTAB protocol mentioned above involving two chloroform extractions and a high-salt isopropanol [14]. DNA was eluted with $30 \mu \mathrm{l}$ water and samples were diluted accordingly to a final concentration of $20 \mathrm{ng} / \mu \mathrm{l}$. DNA samples were quantified using a Nanodrop spectrophotometer (Nyxor Biotech, Paris, France).

\section{S rRNA gene amplicon generation and 454 sequencing}

Multiplexed bacterial tag-encoded FLX amplicon pyrosequencing (bTEFAP) was performed using the Titanium platform (Roche Applied Science, Indianapolis, IN) as previously described [15] in a commercial facility (Research and Testing Laboratories, Lubbock, TX). Briefly, a single step PCR using the primers that span the variable regions V1-V3 of the $16 \mathrm{~S}$ gene, $28 \mathrm{~F} 5^{\prime} \mathrm{GAG}-$ TTTGATGNTGGCTCAG and 519r 5'GTNTTACNGGGG-
CKGCTG), was used to amplify the $16 \mathrm{~S}$ rRNA genes as well as to add adaptor sequences and sample-specific 8-mer oligonucleotide tags (barcodes) to the amplicons. A total of 30 PCR cycles were run using a mixture of HotStart and HotStar high fidelity taq polymerases (Qiagen).

\section{Sequence analysis and statistical analyses}

Sequence analysis was performed using the Quantitative Insights into Microbial Ecology (QIIME) pipeline [16] (version 1.2.0-dev, svn revision 1755) using default parameters unless otherwise noted. Sequences were first screened for quality using the following parameters: minimum quality score of 25 , minimum sequence length of $200 \mathrm{bp}$, maximum length of $1000 \mathrm{bp}$, and no ambiguous bases in the entire sequence or mismatches in the primer sequence. Any sequences not meeting these parameters were excluded from downstream analyses. Sequences were then sorted by barcode into their respective samples and the barcode and primer sequences were removed. The sequences were denoised using the QIIME denoiser [17] and operational taxonomic unites (OTUs) were clustered de novo from the denoised sequences using uclust [18] at 97\% identity. A representative sequence for each OTU was chosen as the centroid of each cluster, and these representative sequences were aligned using PyNAST [16]. A phylogenetic tree was constructed using the FastTree program [19] for use in phylogenetic diversity calculations. Taxonomy was assigned using BLAST against the Silva database (prefiltered at $97 \%$ identity). Chimeras were removed from the reference set on the basis of identification as chimeric via ChimeraSlayer [20]. Organelle sequences were excluded from the downstream analysis by filtering out all of the sequences whose taxonomy assignment contained the text "chloroplast" or "mitochondria".

Weighted and unweighted UniFrac distances were computed between all samples after subsampling all samples to an even depth of 510 sequences per sample to control for differing depths of sequencing across the samples (the minimum, median and maximum sequences per sample, prior to the even sampling, were 179, 16155, and 21698, respectively). Principal Coordinates Analysis (PCoA) was applied to visualize the differences between the sample types. To test whether microbial community differences between sample types (water versus kelp) were significantly greater than differences within sample type (water versus water and kelp versus kelp) we performed a Monte Carlo simulation based on 1000 iterations of shuffling sample labels. All beta diversity results were confirmed at a sampling depth of 5482 sequences per sample, which allowed inclusion of several samples that were initially dropped because their sequence coverage fell below the even sampling depth (data not shown). Alpha diversity was computed using the full data set at a depth of 1031 sequences per sample. The sequence data has been deposited in the European Molecular Biology Laboratory (EMBL) Nucleotide Archive with accession number ERP002019, and can also be found in the QIIME Database under the study id 820 (http://www.microbio.me/quime/).

\section{Results}

\section{Diversity of microbial communities}

After denoising, 276,908 reads were used for the subsequent analyses. The sample libraries ranged from 37,330 to 3,199 reads. The reads were assigned to 4,080 operational taxonomic units (OTUs) at 97\% sequence identity level. The kelp surface libraries had the highest abundance of chloroplast and mitochondrial sequence contamination, ranging from $12-92 \%$ of all the sequences. The total number of bacterial sequences per library before and after the chloroplast and mitochondria removal is listed in 
Table 1. The very low diversity found on the kelp surface in March was due to the dominance of one OTU belonging to a chloroplast (OTU 858). This OTU formed nearly $84 \%$ of the sequences in the kelp surface sample taken in March.

\section{Bacteral community composition on Macrocystis pyrifera and in kelp forest waters}

At the phylum level, kelp surfaces and seawater samples generally had similar community composition (Fig. 1). The seawater samples inside and outside the kelp forest were predominantly composed of Alphaproteobacteria, Gammaproteobacteria, and Bacteroidetes. We found 20 bacterial phyla in at least one sample, with the most abundant groups within the Proteobacteria $(38.1 \%$ of the sequences), Bacteroidetes (22.5\%), Actinobacteria $(9.0 \%)$, Verrucomicrobia (9.0\%) and Planctomycetes (5.3\%). The dominant phylotypes of the kelp surface environment, both abundant and widespread in kelp and/or water samples, were members of the Gamma-, Beta-, and Alphaproteobacteria within the Proteobacteria, and the Bacteroidetes (Table 2). The relative abundances of these dominant OTUs were highly variable between March and April/May samples and between the kelp surface and overlying seawater (Table 2). For instance OTU 1941 represents an uncultured member of the Pseudomonadales that made up more than $30 \%$ of the kelp surface sequences in May but was not detected in any of the seawater samples from any of the times sampled. Similar patterns were observed with other dominant phylotypes including OTU 741 (Burkholderiales order), OTU 6384 (Rhodobacteriales order) and OTU 55 (SAR11 clade) (Tables 2 and 3).

Considerable differences between the water column samples and the kelp surface were evident at finer levels of phylogenetic resolution. At the class level, the differences among the kelp and water samples were significant (one-tailed, two-sample t-test parametric $\mathrm{p}=1.27 \times 10^{-50}$, non-parametric $\left.\mathrm{p}<0.001\right)$. The seawater was dominated by Alphaproteobacteria $(60 \%$ of total sequences), while the kelp surface was dominated by Gammaproteobacteria $(55 \%)$. These differences among samples were also evident at the order level (one-tailed, two-sample t-test parametric $\mathrm{p}=1.27 \times 10^{-50}$, non-parametric $\mathrm{p}<0.001$ ), as seen by the different composition of the main classes within the Proteobacteria (Alpha, Beta and Gamma) in the seawater and kelp surface samples (Fig. 2).

Within the Alphaproteobacteria class (Fig. 2A), SAR11 and Rhodobacteriales were particularly abundant in the water samples. Together, these two groups accounted for $85 \%$ to $95 \%$ of all of the sequences in the water with alternating prevalence of SAR 11 in March and Rhodobacteriales in May. The kelp samples were dominated by the Rhodobacteriales, constituting over $60 \%$ of all the bacterial sequences present (Fig. 2A) and to a lesser extent the Phyllobacteriales that were present only in May. In the seawater, $69 \%$ of the Gammaproteobacteria sequences were

Table 1. Summary of the sequencing results.

\begin{tabular}{|c|c|c|c|c|c|c|c|c|c|}
\hline Date & Sample & Chloroplast & $\%$ & Mitochondria & $\%$ & Bacteria & $\%$ & 97\% OTUs & Chao1 \\
\hline \multirow[t]{6}{*}{ March } & Water in & 4239 & 17.8 & 923 & 3.9 & 18676 & 78.3 & 232 & 600 \\
\hline & & 2502 & 14.5 & 696 & 4.0 & 14009 & 81.4 & 228 & 498 \\
\hline & Water out & 5376 & 19.0 & 1150 & 4.1 & 21698 & 76.9 & 193 & 515 \\
\hline & & 5321 & 22.2 & 1100 & 4.6 & 17538 & 73.2 & 204 & 423 \\
\hline & Kelp & 13321 & 90.0 & 1300 & 8.8 & 179 & 1.2 & 38 & 51 \\
\hline & & 31695 & 84.9 & 5125 & 13.7 & 510 & 1.4 & 62 & 137 \\
\hline \multirow[t]{4}{*}{ April } & Water in & 2654 & 11.1 & 1141 & 4.8 & 20117 & 84.1 & 204 & 325 \\
\hline & & 2828 & 11.6 & 1288 & 5.3 & 20289 & 83.1 & 199 & 383 \\
\hline & Water out & 209 & 3.5 & 276 & 4.6 & 5482 & 91.9 & 154 & 213 \\
\hline & & 944 & 5.2 & 861 & 4.7 & 16382 & 90.1 & 207 & 308 \\
\hline \multirow[t]{6}{*}{ May } & Water in $0 \mathrm{~m}$ & 157582 & 92.6 & 460 & 0.3 & 12148 & 7.1 & 238 & 553 \\
\hline & & 5020 & 21.1 & 727 & 3.1 & 18056 & 75.9 & 256 & 912 \\
\hline & Water out $0 \mathrm{~m}$ & 2065 & 10.3 & 680 & 3.4 & 17292 & 86.3 & 243 & 710 \\
\hline & & 2223 & 11.9 & 589 & 3.1 & 15928 & 85.0 & 263 & 783 \\
\hline & Kelp 0m & 12550 & 63.0 & 1394 & 7.0 & 5990 & 30.0 & 98 & 154 \\
\hline & & 4518 & 76.0 & 295 & 5.0 & 1130 & 19.0 & 144 & 174 \\
\hline \multirow[t]{6}{*}{ May } & Water in $10 \mathrm{~m}$ & 4013 & 19.5 & 661 & 3.2 & 15855 & 77.2 & 243 & 565 \\
\hline & & 2420 & 10.8 & 791 & 3.5 & 19129 & 85.6 & 265 & 778 \\
\hline & Water out $10 \mathrm{~m}$ & 2617 & 12.7 & 538 & 2.6 & 17483 & 84.7 & 240 & 650 \\
\hline & & 2655 & 12.9 & 459 & 2.2 & 17462 & 84.9 & 230 & 644 \\
\hline & Kelp 10m & 5090 & 33.7 & 489 & 7.4 & 1031 & 15.6 & 94 & 125 \\
\hline & & 2228 & 69.6 & 447 & 14.0 & 524 & 16.4 & 45 & 49 \\
\hline
\end{tabular}

Abbreviations: OTU, operational taxonomic units

Water In samples were taken in close proximity to the kelp, inside the kelp forest; Water Out samples were sampled outside the kelp forest perimeter. The number of reads and the percentage of total sequences are displayed, categorized as being bacterial or organelle derived. OTUs at $97 \%$ identity and Chao 1 OTU richness were estimated after sub sampling all samples to an even depth of 1031 sequences per sample to control for differing depths of sequencing across the samples (the minimum, median and maximum sequences per sample, prior to the even sampling, were 179, 16155, and 21698, respectively), and after removing organelle-derived sequences. Sampled depth for all samples and dates was 1 meter, unless otherwise noted.

doi:10.1371/journal.pone.0067480.t001 


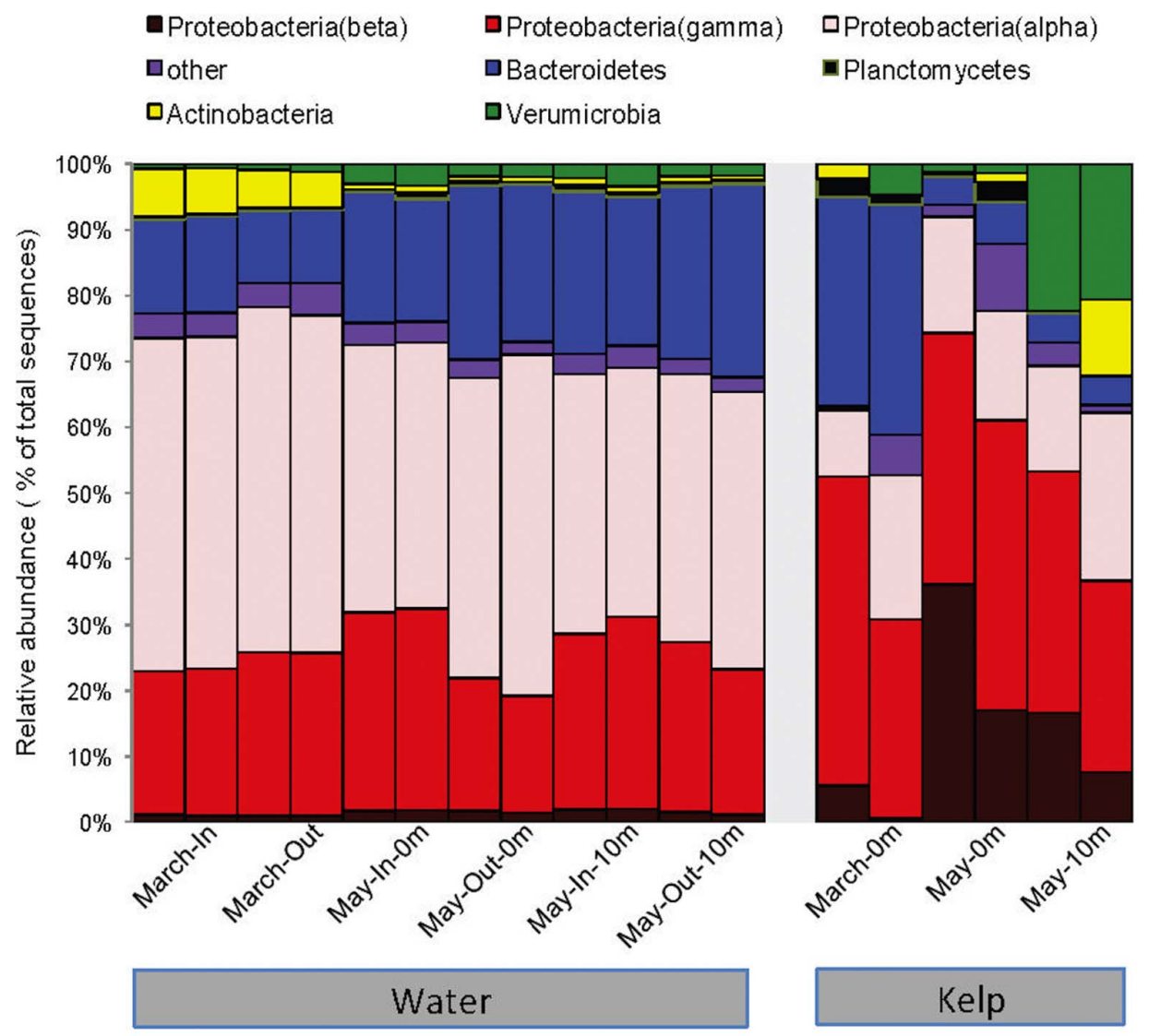

Figure 1. Kelp surface and seawater bacterial communities at the level of phyla. "Kelp" refers to the kelp surface samples; "Water-In" refers to the seawater samples adjacent to the kelp sampled; "Water-Out" refers to the seawater sampled outside the perimeter of the kelp forest. doi:10.1371/journal.pone.0067480.g001

assigned to members of the order Oceanospiralles, which was not a major constituent of the kelp surface community $(2 \%$ of all kelp sequences; Fig. 2B). Alteromonadales was the next most frequent order in the water samples, but was also rare in the kelp surface community. However, Pseudomonadales and Chromatiales dominated the communities on kelp samples, followed by a large number of uncultured Gammaproteobacteria sequences $(8 \%$ of all kelp sequences). These two orders were rare in the seawater community $(2 \%$ of all sequences in May). The third most abundant class of the Proteobacteria, Betaproteobacteria, was composed of the orders Methylophilales and Burkholderiales. The order Nitrosomonadales was also present, but it was much less abundant and only found in the seawater (Fig. 2C). Methylophiliales sequences dominated inshore and offshore seawater samples,

Table 2. Most dominant OTUs in all sites sampled for this study and their average relative abundances (as percentages of all sample 16S rRNA gene sequences recovered).

\begin{tabular}{|c|c|c|c|c|c|c|c|c|}
\hline Water March & Water May & Kelp March & Kelp May & OTU\# & Nearest neighbor & $\%$ ID & Class & Order \\
\hline NA & NA & 2 & 31.3 & 741 & Aquabacterium & 91 & Betaproteobacteria & Burkholderiales \\
\hline NA & NA & 2.4 & 17.3 & 3619 & Verrucomicrobiaceae & 100 & Verrucomicrobiae & Verrucomicrobiales \\
\hline NA & NA & NA & 31.9 & 1941 & Pseudomonas & 100 & Gammaproteobacteria & Pseudomonadales \\
\hline NA & NA & 10.3 & 0.7 & 3882 & Flavobacteriaceae & 100 & Flavobacteria & Flavobacteriales \\
\hline NA & NA & 4.6 & 1.4 & 3991 & Stenotrophomonas & 99 & Gammaproteobacteria & Xanthomonadales \\
\hline 2.7 & 1.6 & 0.1 & 0.0 & 5417 & Ulvibacte & 100 & Flavobacteria & Flavobacteriales \\
\hline 5.2 & 28.3 & 0.6 & 0.6 & 6384 & Thalassobacter & 83 & Alphaproteobacteria & Rhodobacterales \\
\hline 5.0 & 12.6 & 0.1 & 0.1 & 4492 & Oleiphilaceae & 87 & Gammaproteobacteria & Oceanospirillales \\
\hline 32.1 & 9.2 & NA & NA & 55 & Pelagibacter & 100 & Alphaproteobacteria & SAR11 \\
\hline
\end{tabular}

Abbreviations: OTU, operational taxonomic units; Phylogenetic classification was determined by BLAST against the Silva database

OTUs were considered dominant if they were both highly abundant and occurred frequently in kelp samples. "NA" indicates that the OTU was not included within the 10 most dominant for that sample.

doi:10.1371/journal.pone.0067480.t002 
Table 3. OTUs at $97 \%$ similarity, which were found in all kelp surface samples, presented as their contribution to the whole community (\% of the total bacterial sequences).

\begin{tabular}{lllll}
\hline Taxon & Classification & March & May-0m & May-10m \\
\hline Alphaproteobacteria & Sphingomonadaceae & $12.7 \%$ & $2.1 \%$ & $1.8 \%$ \\
Alphaproteobacteria & Rhizobiales & $12.7 \%$ & $7.0 \%$ & $3.1 \%$ \\
Betaproteobacteria & Burkholderiales & $3.1 \%$ & $26.2 \%$ & $2.6 \%$ \\
Gammaproteobacteria & Pseudomonadales & $8.1 \%$ & $30.4 \%$ & $38.4 \%$ \\
Gammaproteobacteria & Group2 & $8.3 \%$ & $1.1 \%$ & $0.5 \%$ \\
Gammaproteobacteria & Chromatiales & $8.2 \%$ & $2.0 \%$ & $4.6 \%$ \\
Bacteroidetes & Flavobacteriaceae & $18.9 \%$ & $3.1 \%$ & $1.8 \%$ \\
\cline { 1 - 2 } & TOTAL & $71.96 \%$ & $71.86 \%$ & $52.74 \%$ \\
\hline
\end{tabular}

Abbreviations: OTU, operational taxonomic units; Classification indicates the taxonomical affiliation of the OTU sequences, and the level of taxonomic classification chosen included at least $99 \%$ of all sequences for a particular OTU. doi:10.1371/journal.pone.0067480.t003

while the kelp surface samples were almost entirely composed of OTUs from the Burkholderiales order, with very little variability between the surface and deep samples.

\section{Structure of kelp forest bacterial communities}

Principal Coordinates Analysis (PCoA) of unweighted UniFrac distances at exactly 510 sequence per sample show that the kelp surface and seawater communities harbour characteristic communities of Bacteria that differ from one another, evident by their independent clustering on the first principal coordinate axis (Fig. 3). This difference in community composition is statistically significant: the within-category distances (water-to-water and kelpto-kelp) were significantly smaller than the between-category distances (water-to-kelp) when compared with both parametric and non-parametric t-tests (one-tailed, two-sample t-test parametric $\mathrm{p}$-value $=1.27 \times 10^{-50}$, Monte Carlo t-test $\mathrm{p}$-value $<0.001$ ).

Differences among bacterial communities were also assessed using weighted UniFrac, which takes into account the relative abundance of each OTU rather than presence/absence alone (Fig. 4). As with unweighed UniFrac, the between-sample-type distances (water-to-kelp) were significantly higher that the withinsample-type distances (Fig. 4A; one-tailed, two-sample t-test parametric p-value $=6.49 \times 10^{-14}$, Monte Carlo p-value $<0.001$ ) . A weighed UniFrac PCoA plot showing the samples colored by month of sampling (Fig. 4B) suggests that there may be seasonal patterns in the community composition of both kelp surfaces and surrounding sea water (which has previously been shown in sea water [21].

The data suggest that at least two factors affected community clustering: the sample type and the month of sampling. Along the PC1 axis (Fig. 4, 51.72\% of the variance explained), the samples segregated by type. On PC2 (22.82\% of the variance explained) we observe clustering of the water samples by sampling date: the March and April/May samples were the most different, although subtle differences occured between the April and May water samples. Shifts over time in the kelp samples were difficult to discern because the sampling occurred only in March and May, and in addition, one of the March samples had too few bacterial sequences (179) to be included in the analyses. Seasonal differences may thus occur in the kelp habitat, although a more detailed longitudinal study would be needed in order to demonstrate these differences conclusively.

\section{Discussion}

\section{Seawater-associated communities}

The community structure of bacterioplankton in the Monterey Bay region is well studied, with available data on the spatiotemporal structure of microbial populations and their response to episodic hydrodynamic events [22-25]. Our results from sequencing the 16S RNA gene from seawater samples inside and outside the kelp forest are in agreement with the published literature of coastal prokaryotic planktonic communities being dominated by Alphaproteobacteria, Gammaproteobacteria, Bacteroidetes, Actinobacteria and Betaproteobacteria [26,27].

The most abundant OTUs from our seawater samples belong to known groups of bacteria characteristic of the surrounding area such as the Alphaproteobacteria SAR11, the Gammaproteobacteria from the Roseobacter clade and the Betaproteobacteria Methylophilales (Fig. 2A, B, G). One of the most abundant groups of Gammaproteobacteria we observed was the Oceanospirillales, which made up about $40 \%$ of the entire seawater community (Fig. 2A). The distribution of SAR11-related phylotypes in waters around the kelp forest with highest abundance in March, and much lower abundances in May (Table 2). We did not detect any seasonal changes in the predominance of the Betaproteobacteria or Gammaproteobacteria phylotypes in our seawater samples, and their distribution was constant throughout the study (Fig. 2B and G). Overall, the communities in both inshore and offshore water samples from the Monterey Bay kelp forest were identified as being typically marine-like.

\section{Dominant bacterial taxa associated with M. pyrifera surface}

As in recent studies of surface-associated bacterial communities on sponges and macroalgae [7], the kelp and seawater communities were similar at the phylum level, with the both the water and kelp samples dominated by Proteobacteria (predominantly Alphaproteobacteria) and Bacteroidetes. The kelp surface libraries were characterized by sequences from the Rhodobacteraceae, Sphingomonadaceae (Alphaproteobacteria), Flavobacteraceae and Saprospiraceae (Bacteroidetes) families and included sequences from the Verrumicrobia and unclassified Gammaproteobacteria. These observations are in broad agreement with the relatively limited data regarding marine kelp-associated bacterial communities[11,12,28,29].

The differences in the kelp and water community compositions may be due to the different nature of the physical environments harboring the two communities. The composition of the bacterial community can presumably be influenced by the chemistry of the kelp's surface, where metabolites and tissue composition can attract or repel certain bacteria resulting in communities comprised of bacterial groups adapted to kelp-surface lifestyles [30]. Kelp metabolites affect bacterial growth and attachment, and therefore, the composition of the bacterial community is influenced to some extent by the surface chemistry of the kelp. Metabolites and surface tissue composition may selectively attract or repel bacteria with patterns driven by the temporally variable nature of kelp exudates [4] and thereby shaping the microbial composition on its surface[31,32].

It is known that kelp tissue concentrations of mannitol and laminarin increase during the winter and early spring, when growth rates are low, and are reduced (presumably to support growth) during the late spring and summer, when the onset of upwelling brings about high kelp growth rates [33]. Within the Alphaproteobacteria, Rhizobiales and/or Rhodobacterales were consistently found on kelp surfaces both in the March and April/ 
A.
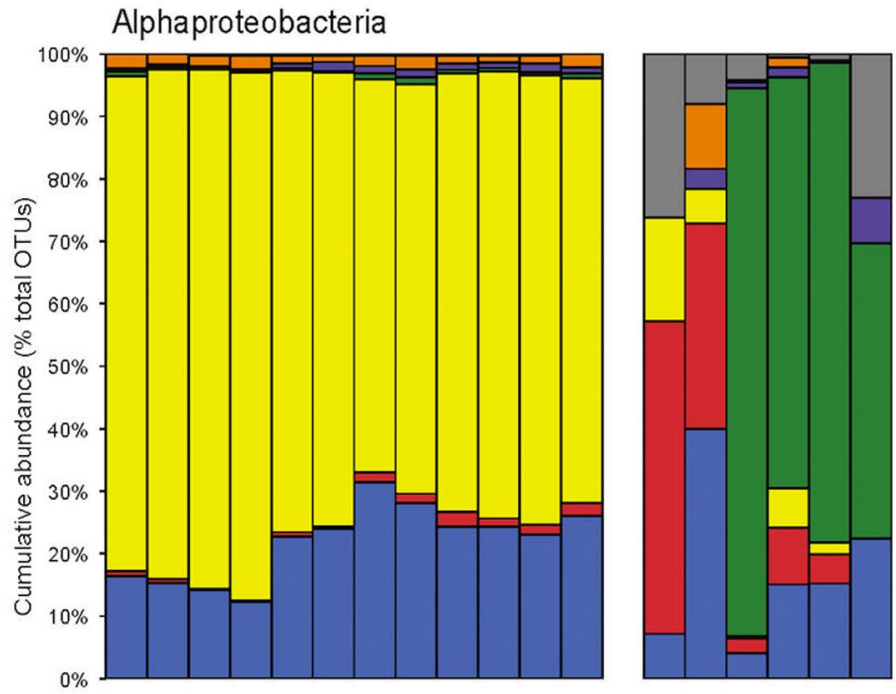

口Xanthomonadales

口Thiotrichales

- Vibrionales

口Pseudomonadales

口Oceanospirillales

口Chromatiales

口Alteromonadales

B.

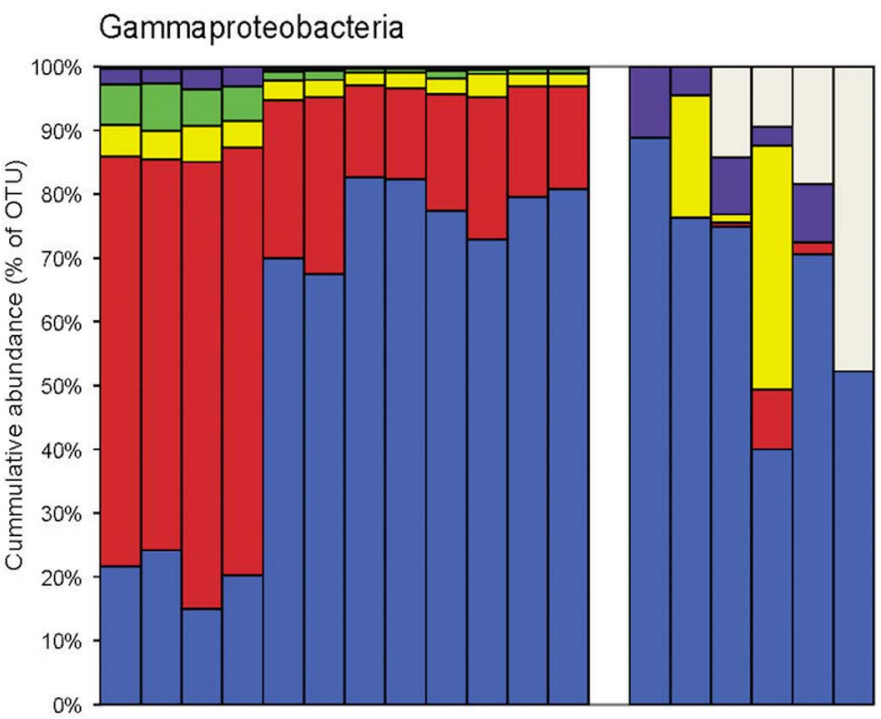

口Phyllobacteriaceae

-Sphingomonadaceae

口Other

口uncultured

-SAR11

口Rhodobacteraceae

C.
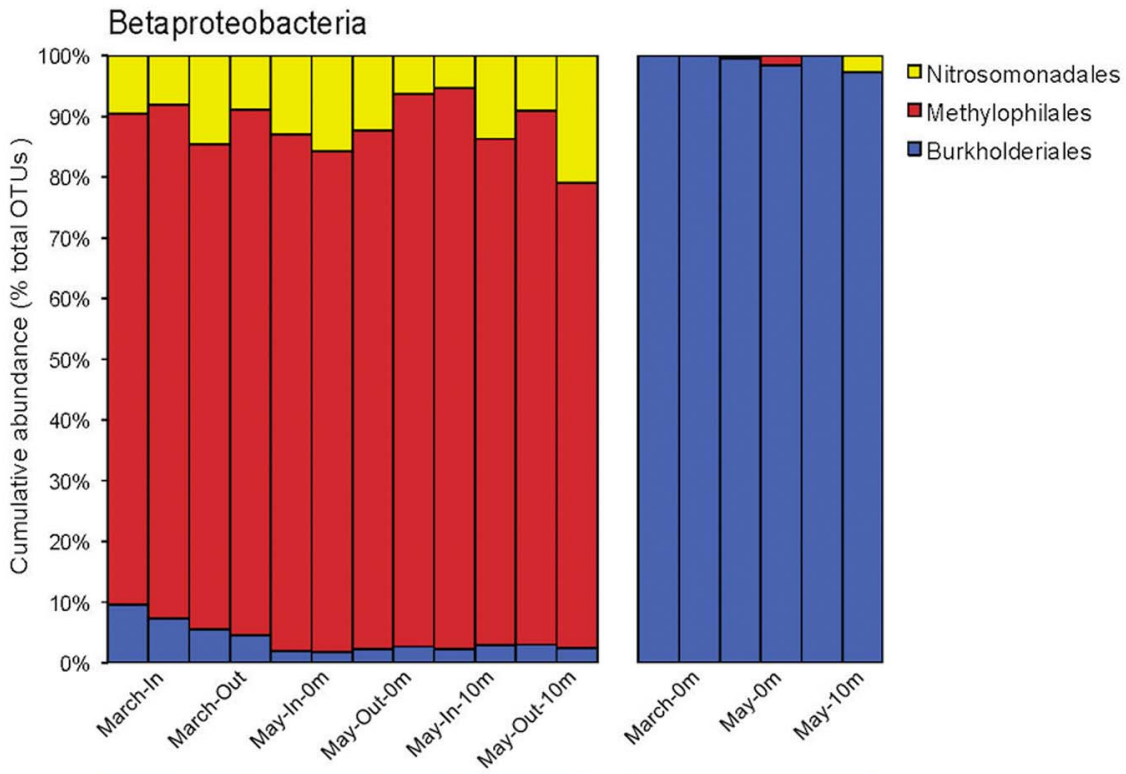

Water

Kelp 
Figure 2. Bacterial distribution of the most abundant groups within the predominant classes of the Proteobacterial phylum. The Alpha- (A), Gamma- (B) and Betaproteobacteria (C) were consistently present in high abundances in all of the samples. A further evaluation within these classes showed differences in the profiles and abundance of the bacterial groups in seawater and on the kelp surface. X-axis sample designation is the same as Figure 1.

doi:10.1371/journal.pone.0067480.g002

May samples. OTUs closely related to sequences within the Rhizobiales comprised $10 \%$ and $9 \%$ of the March and May communities on the kelp surface, respectively. These bacterial groups are known for their antibacterial activity, suggesting that the kelp/bacteria symbiosis may be mutualistic with these groups assisting in defense of the kelp against potential pathogens. [34]

We expect bacteria that are capable of utilizing the dissolved carbon that is exuded from kelp cells as well as components of the extracellular mucus might be found on kelp surfaces. For example, the fucoidan-degrading activity of Verrucomicrobia may explain the abundance of this phylum on F. vesiculosus [35]. Similarly, Sphingomonodaceae, found in our study, have fucoidanolytic, alginolytic and polycyclic aromatic hydrocarbon (PAH)-degrading activities and may benefit from the kelp's surface components [3639]. Bacteria belonging to the Bacteroidetes, Sphingobacteria and Actinobacteria have agarolytic and carrageenanolytic properties and may thus be attracted to cell-wall components of the kelp [40]. The March kelp surface harbored bacteria belonging to the Flavobacteriales, Sphingobacteriales, Xanthomonadales and Chromatiales. Bacteria belonging to the Bacteroidetes have been found to degrade complex polysaccharides, and Flavobacteriaceae strains have been isolated from rotting kelp, which may have similar characteristics to the kelp fronds from our March samples [41]. It is possible that the relatively high abundance of this group in such samples is linked to agarase-production, which could enable these bacteria to utilize agar from kelp fronds and benefit from exudates from old and damaged kelp tissue [37,38,40]. These kelp-surface associated bacteria may therefore represent an opportunistic collection of phylotypes that can colonize kelp tissue.

\section{Structure of kelp forest seawater bacterial communities}

We found diverse sequence clustering patterns in the kelp and seawater samples examined, suggesting that bacterial community in the water column is distinct from the communities on the surface of the kelp. Although the seawater and kelp surface communities shared similarities at the phylum level, their bacterial communities were strikingly distinct at lower taxonomic levels. At the $97 \%$ OTU level, less than $2 \%$ of OTUs occurred in both the seawater and surface of the kelp.

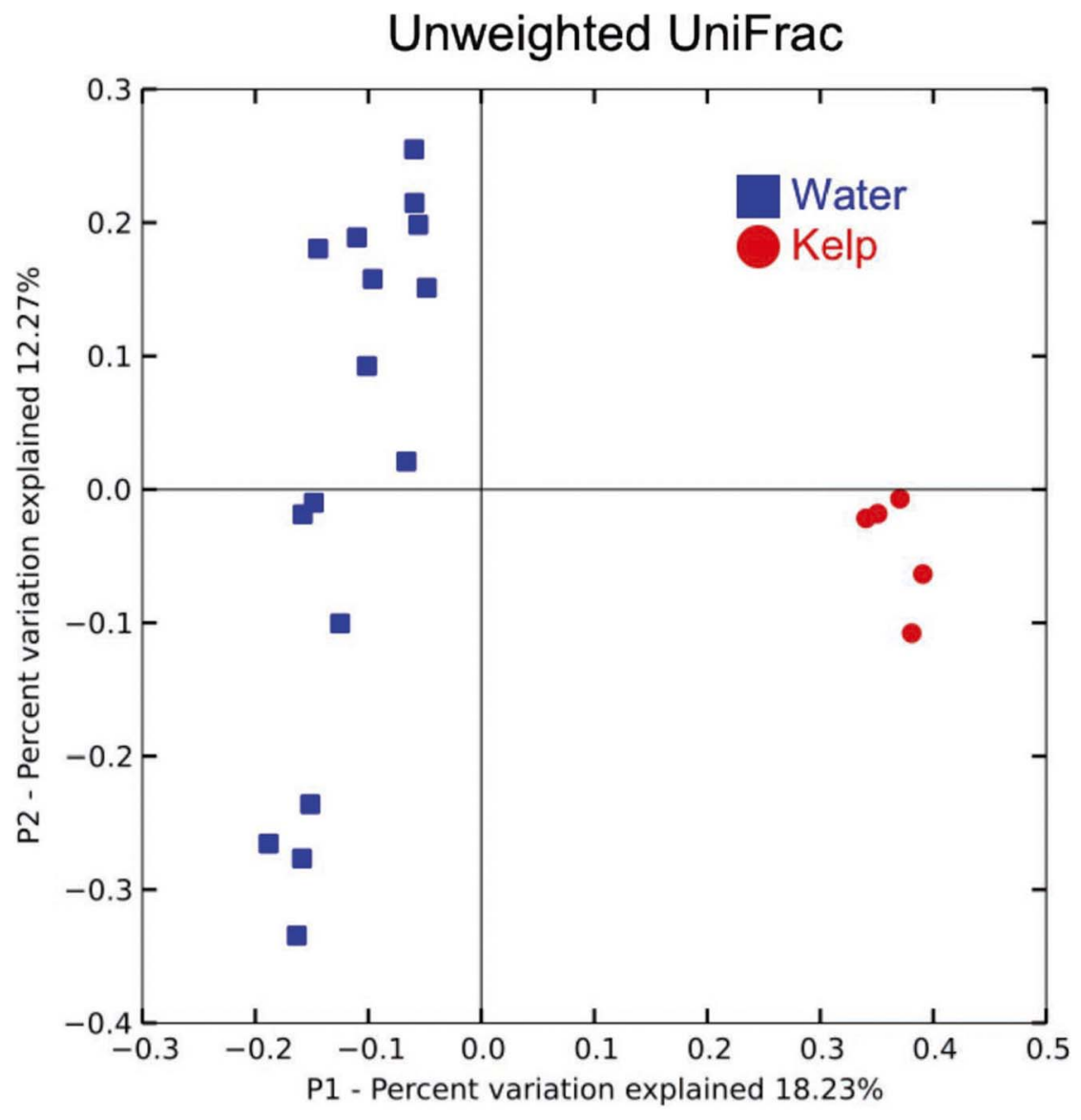

Figure 3. Unweighted UniFrac principal coordinates analysis (PCOA) plots computed at exactly 510 sequences/sample illustrate the relationship between sample type (blue $=$ water, red $=$ kelp) community similarities. Percentages of variance explained by each principal coordinate (P1 and $\mathrm{P} 2)$ are shown on the $\mathrm{x}$ - and $y$-axes.

doi:10.1371/journal.pone.0067480.g003 
A.

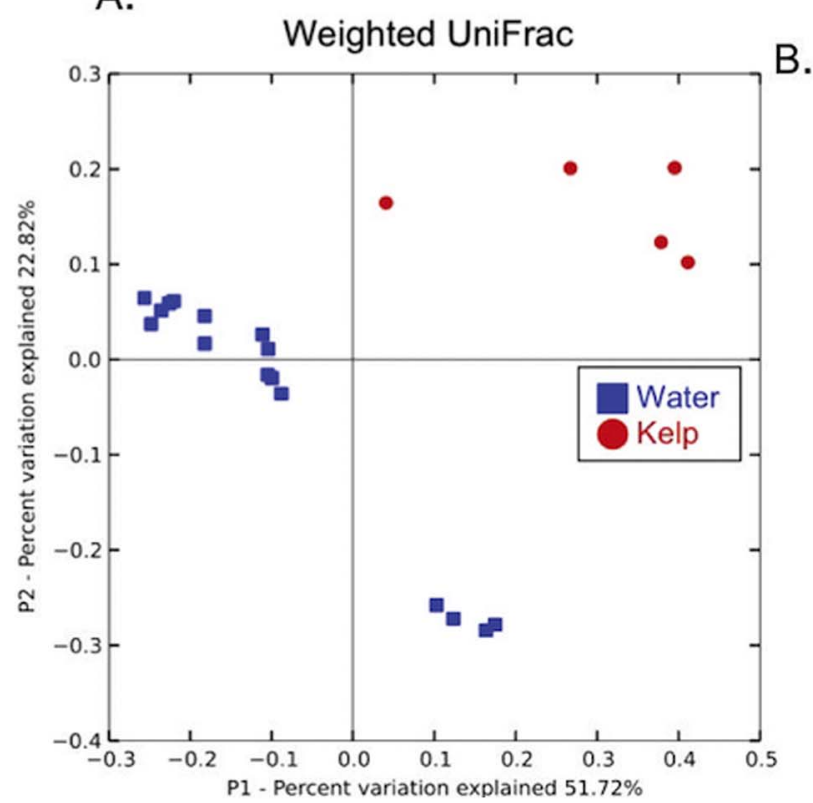

B.

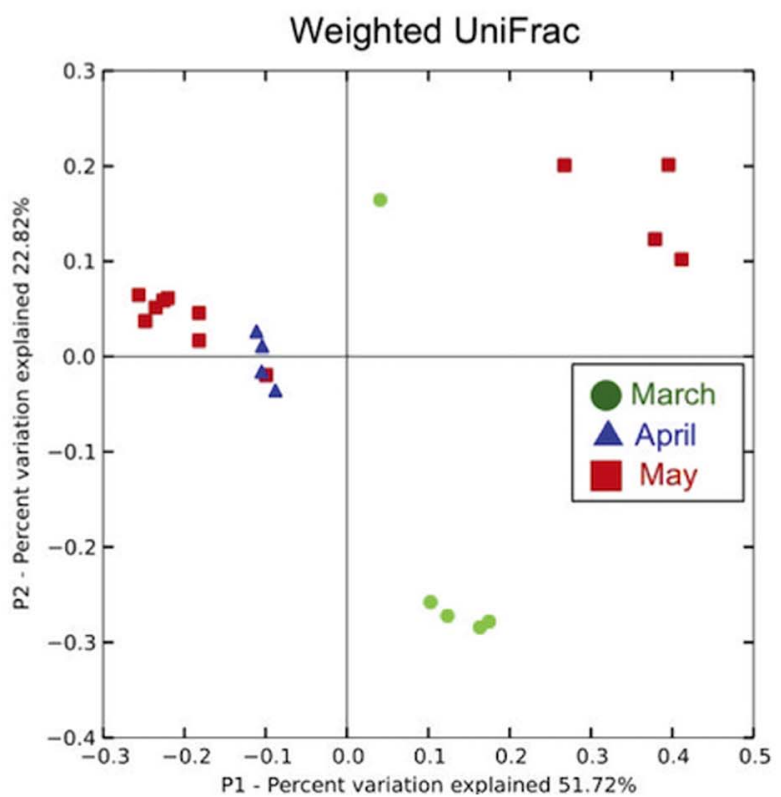

Figure 4. Weighed UniFrac principal coordinates analysis (PCoA) computed at exactly 510 sequences/sample illustrate the influence of sample type (Fig. 4A) and sample collection date (Fig. 4B). The coloring reflects the different dates on which the samples were taken (green $=$ March, blue $=$ April, red = May). "Kelp" refers to the kelp surface samples; "Water" refers to the seawater samples adjacent to the kelp sampled as well as those sampled outside the perimeter of the kelp forest. Percentages of variance explained by each principal coordinate (P1 and $\mathrm{P} 2$ ) are shown on the $\mathrm{x}$ - and $\mathrm{y}$-axes.

doi:10.1371/journal.pone.0067480.g004

We additionally observed temporal variation in the bacterial communities both on the surface of the kelp and in the water column. The relative abundances of the dominant bacteria from kelp surface samples changed between March and May (e.g., Gammaproteobacteria) (Table 2), but the abundances of other taxa (e.g. Alphaproteobacteria (Rhizobiales)) were largely unaffected by sampling time, indicating that the abundances of the latter groups may be influenced by other factors. Specific bacteria within Pseudomonadales and Burkholderiales were more abundant in May kelp samples, and as a result the entire surface community contained fewer members at this time. March kelp samples were more diverse, with 8 OTUs making up the majority of the surface community, whereas only three OTUs accounted for $81 \%$ of the kelp surface community in May (Table 3). We note that it is not possible to draw conclusions about seasonality of these communities since we sampled over only one three month period.

Seawater samples from inside and outside the kelp forest were similar in bacterial community composition during our study. Our sampling sites were close relative to the known dispersal distance for local waters $(>4 \mathrm{~km})$ [42]. Since our sampling stations were less than 4 kilometers apart, it is not surprising that we found similar communities inside and outside the kelp forest. However, bacteria associated with the kelp surface exhibited a different pattern in community composition harboring different bacterial communities from those present in the water. The kelp surface may thus be acting as a highly specialized habitat for microbes distinct from that of the surrounding water column, selecting for growth of microbes that could be present in the water but perhaps at in very

\section{References}

1. Wahl M (2008) Ecological lever and interface ecology: epibiosis modulates the interactions between host and environment. Biofouling: The Journal of Bioadhesion and Biofilm Research 24: 427-438. low numbers and that are able to thrive once they colonize the algal tissue.

In conclusion, we found several strong microbial community clustering patterns in the kelp and seawater samples examined, suggesting a bacterial community in the water column distinct from the communities on the surface of the kelp. Furthermore, bacteria associated with the kelp surface from the same locations sampled at different times exhibited different community compositions, suggesting temporal variation in kelp-associated microbial communities. Notwithstanding such dissimilarities, the communities in all the samples were typical of other marine environments. Future studies should address whether seasonal changes in environmental conditions, including temperature and nutrient load, affect community composition on the surface of kelp.

\section{Acknowledgments}

We thank Dr. Stephen Litvin, Dr. Sarah Lee and Paul Leary for help with data collection. We acknowledge the help of the staff at Hopkins Marine Station, especially Chris Patton, during fieldwork, and the Gordon and Betty Moore Foundation for the use of the Microbial Environmental Genomics: Applications, Modeling, Experimentation, and Remote Sensing (MEGAMER) facility and John Zehr for his support and advice.

\section{Author Contributions}

Conceived and designed the experiments: VKM SRP. Performed the experiments: VKM. Analyzed the data: VKM JGC. Contributed reagents/ materials/analysis tools: VKM SRP JGC RK. Wrote the paper: VKM. Statistical expertise: JGG. 
3. Bengtsson MM, Sjotun K, Lanzen A, Ovreas L (2012) Bacterial diversity in relation to secondary production and succession on surfaces of the kelp Laminaria hyperborea. ISME J.

4. Colleen J, Davison IR (2001) Seasonality and thermal acclimation of reactive oxygen metabolism in Fucus vesiculosus (phaeophyceae). Journal of Phycology 37: $474-481$

5. Taylor MW, Schupp PJ, Dahllof I, Kjelleberg S, Steinberg PD (2004) Host specificity in marine sponge-associated bacteria, and potential implications for marine microbial diversity. Environmental Microbiology 6: 121-130.

6. Reis AMM, Araujo SD, Moura RL, Francini RB, Pappas G, et al. (2009) Bacterial diversity associated with the Brazilian endemic reef coral Mussismilia braziliensis. Journal of Applied Microbiology 106: 1378-1387.

7. Longford SR, Tujula NA, Crocetti GR, Holmes AJ, Holmstrom C, et al. (2007) Comparisons of diversity of bacterial communities associated with three sessile marine eukaryotes. Aquatic Microbial Ecology 48: 217-229.

8. Bulleri F, Benedetti-Cecchi L, Acunto S, Cinelli F, Hawkins SJ (2002) The influence of canopy algae on vertical patterns of distribution of low-shore assemblages on rocky coasts in the northwest Mediterranean. Journal of Experimental Marine Biology and Ecology 267: 89-106.

9. Linley EAS, Field JG (1982) The nature and ecological significance of bacterial aggregation in a nearshore upwelling ecosystem. Estuarine, Coastal and Shelf Science 14: 1-11.

10. Graham MH, Kinlan BP, Druehl LD, Garske LE, Banks S (2007) Deep-water kelp refugia as potential hotspots of tropical marine diversity and productivity. Proceedings of the National Academy of Sciences 104: 16576-16580.

11. Staufenberger T, Thiel V, Wiese J, Imhoff JF (2008) Phylogenetic analysis of bacteria associated with Laminaria saccharina. Fems Microbiology Ecology 64: 65-77.

12. Bengtsson MM (2011) Bacterial biofilms on the kelp, Laminaria hyperborea [Thesis (Ph.D.)]. Bergen [Norway]: University of Bergen. 1 v. (various pagings) p.

13. Burke C, Kjelleberg S, Thomas T (2009) Selective Extraction of Bacterial DNA from the Surfaces of Macroalgae. Applied and Environmental Microbiology 75: $252-256$.

14. Dempster EL, Pryor KV, Francis D, Young JE, Rogers HJ (1999) Rapid DNA extraction from ferns for PCR-based analyses. Biotechniques 27: 66-68.

15. Dowd SE, Sun Y, Wolcott RD, Domingo A, Carroll JA (2008) Bacterial TagEncoded FLX Amplicon Pyrosequencing (bTEFAP) for Microbiome Studies: Bacterial Diversity in the Ileum of Newly Weaned Salmonella-Infected Pigs. Foodborne Pathogens and Disease 5: 459-472.

16. Caporaso J, Kuczynski J, Stombaugh J, Bittinger K, Bushman F, et al. (2010) QIIME allows analysis of high-throughput community sequencing data. Nat Methods 7: 335-336.

17. Reeder J, Knight R (2010) Rapidly denoising pyrosequencing amplicon reads by exploiting rank-abundance distributions. Nat Meth 7: 668-669.

18. Edgar RC (2010) Search and clustering orders of magnitude faster than BLAST. Bioinformatics 26: 2460-2461.

19. Price MN, Dehal PS, Arkin AP (2010) FastTree 2 'Approximately MaximumLikelihood Trees for Large Alignments. PLoS ONE 5: e9490.

20. Haas BJ, Gevers D, Earl AM, Feldgarden M, Ward DV, et al. (2011) Chimeric 16S rRNA sequence formation and detection in Sanger and 454-pyrosequenced PCR amplicons. Genome Res 21: 494-504.

21. Gilbert JA, Steele JA, Caporaso JG, Steinbruck L, Reeder J, et al. (2012) Defining seasonal marine microbial community dynamics. ISME J 6: 298-308.

22. Ottesen EA, Marin R, 3rd, Preston CM, Young CR, Ryan JP, et al. (2011) Metatranscriptomic analysis of autonomously collected and preserved marine bacterioplankton. ISME J.

23. Rich VI, Pham VD, Eppley J, Shi Y, DeLong EF (2011) Time-series analyses of Monterey Bay coastal microbial picoplankton using a 'genome proxy' microarray. Environmental Microbiology 13: 116-134.
24. Suzuki MT, Preston CM, Beja O, de L, Steward GF, et al. (2004) Phylogenetic screening of ribosomal RNA gene-containing clones in bacterial artificial chromosome (BAC) libraries fromdifferent depths in Monterey Bay. Microbial Ecology 48: 473-488.

25. Suzuki MT, Preston GM, Chavez FP, DeLong EF (2001) Quantitative mapping of bacterioplankton populations in seawater: field tests across an upwelling plume in Monterey Bay. Aquatic Microbial Ecology 24: 117-127.

26. Rusch DB, Halpern AL, Sutton G, Heidelberg KB, Williamson S, et al. (2007) The Sorcerer II Global Ocean Sampling Expedition: Northwest Atlantic through Eastern Tropical Pacific. PLoS Biology 5: e77.

27. Rappe MS, Vergin K, Giovannoni SJ (2000) Phylogenetic comparisons of a coastal bacterioplankton community with its counterparts in open ocean and freshwater systems. FEMS Microbiology Ecology 33: 219-232.

28. Burke C, Thomas T, Lewis M, Steinberg P, Kjelleberg S (2010) Composition, uniqueness and variability of the epiphytic bacterial community of the green alga Ulva australis. ISME J.

29. Lachnit T, Blumel M, Imhoff JF, Wahl M (2009) Specific epibacterial communities on macroalgae: phylogeny matters more than habitat. Aquatic Biology 5: 181-186.

30. Zheng L, Han XT, Chen HM, Lin W, Yan XJ (2005) Marine bacteria associated with marine macroorganisms: the potential antimicrobial resources. Annals of Microbiology 55: 119-124.

31. Steinberg PD, De Nys R (2002) Chemical mediation of colonization of seaweed surfaces. Journal of Phycology 38: 621-629.

32. Sapp M, Wichels A, Wiltshire K, Gerdts G (2007) Bacterial community dynamics during the winter-spring transition in the North Sea. FEMS Microbiol Ecol 59: 622-637.

33. Zimmerman RC, Kremer JN (1986) Insitu growth and chemical-composition of the giant-kelp, macrocystis-pyrifera-response to temporal changes in ambient nutrient availability. Marine Ecology-Progress Series 27: 277-285.

34. Rao D, Webb JS, Holmstrom C, Case R, Low A, et al. (2007) Low densities of epiphytic bacteria from the marine alga Ulva australis inhibit settlement of fouling organisms. Applied and Environmental Microbiology 73: 7844-7852.

35. Sakai T, Kawai T, Kato I (2004) Isolation and characterization of a fucoidandegrading marine bacterial strain and its fucoidanase. Marine Biotechnology 6 : $335-346$.

36. Abarzua S, Jakubowski S, Eckert S, Fuchs P (1999) Biotechnological investigation for the prevention of marine biofouling II. Blue-green algae as potential producers of biogenic agents for the growth inhibition of microfouling organisms. Botanica Marina 42: 459-465.

37. Cunliffe M, Kertesz MA (2006) Autecological properties of soil sphingomonads involved in the degradation of polycyclic aromatic hydrocarbons. Applied Microbiology and Biotechnology 72: 1083-1089.

38. Wong TY, Preston LA, Schiller NL (2000) Alginate lyase: Review of major sources and enzyme characteristics, structure-function analysis, biological roles, and applications. Annual Review of Microbiology 54: 289-340.

39. Woo-Jung K, Kim SM, Lee YH, Kim HG, Kim HK, et al. (2008) Isolation and characterization of marine bacterial strain degrading fucoidan from Korean Undaria pinnatifida sporophylls. Journal of Microbiology and Biotechnology 18: 616-623.

40. Michel G, Nyval-Collen P, Barbeyron T, Czjzek M, Helbert W (2006) Bioconversion of red seaweed galactans: a focus on bacterial agarases and carrageenases. Applied Microbiology and Biotechnology 71: 23-33.

41. Goecke F, Labes A, Wiese J, Imhoff JF (2010) Chemical interactions between marine macroalgae and bacteria. Marine Ecology-Progress Series 409: 267-299.

42. Davis CL, Koop K, Muir DG, Robb FT (1983) Bacterial diversity in adjacent kelp-dominated ecosystems. Marine Ecology-Progress Series 13: 115-119. 